P. J. Preinfalk

Dj. Djawari

\section{Atypische Mykobakteriose unter dem klinischen Bild einer Tuberculosis subcutanea et fistulosa}

Atypical Mycobacteriosis Imitating Tuberculosis Subcutanea et Fistulosa

\section{Zusammenfassung}

Eine 42-jährige Patientin war wegen Unterleibsschmerzen infolge eines Thailandaufenthaltes bei V.a. Genitalmykose wiederholt ambulant und stationär behandelt worden. Im Verlauf traten brettharte, fistulierende, druckschmerzhafte, subkutane Knoten auf. Die stationäre Aufnahme bei uns erfolgte unter dem V.a. Aktinomykose. Labor und paraklinische Untersuchungen ergaben im Wesentlichen unauffällige Befunde, die Histologie ließ am ehesten an eine Tuberkulose denken. Der Nachweis von Drusen gelang ebenso wenig wie der säurefester Stäbchen. Das nachgewiesene Mykobakterium chelonae wurde vom nationalen Referenzzentrum Borstel als klinisch nicht relevant eingestuft. Unter hochdosierter Penicillingabe und topischer Therapie mit Ichthyol kam es jedoch zu einer Befundbesserung, so dass wir rückblickend von einer atypischen Mykobakteriose unter dem klinischen Bild einer Tuberculosis subcutanea et fistulosa ausgehen. Die Besserung des klinischen Befundes könnte möglicherweise auf eine für M. chelonae beschriebene Spontanheilung zurückzuführen sein.
Abstract

A 42year old woman had been treated several times because of lower abdominal pain both on an out- and inpatient basis with the presumption diagnosis of a genital mycosis. In the course hardly indurated, fistulous, subcutaneous nodules occurred with tenderness. We hospitalized the patient under the presumption of actinomycosis. The laboratory and paraclinical findings were incospicuous, the histopathologic results corresponded to cutaneous tuberculosis. Neither drusen nor acid-resisting rod-shaped bacteria could be proved. The only found Mycobacterium chelonae was classified as non pathologic by the National Reference Centre Borstel. The efflorescences regressed under high-dose therapy with penicillin and topical application of ichthyol. We assume that the patient suffered from an atypical mycobacteriosis $($ MOTT $=$ Mycobacteria other than tuberculosis) under the clinical picture of a tuberculosis subcutanea and fistulosa. The recovery might be based on a spontaneous regression which is described in literature for M. chelonae in single cases.

\section{Einleitung}

Atypische Mykobakteriosen können unter einem breiten Spektrum unterschiedlicher klinischer Bilder in Erscheinung treten. Die möglichen Infektionswege sind mannigfaltig und die klinischen, mikrobiologischen und histologischen Befunde mitunter irreführend [1,2].
Aufgrund weltweit steigender Zahlen an iatrogenen Infektionen [3-6] sollten sie zunehmend bei differenzialdiagnostischen Erwägungen in Betracht gezogen werden.

Angesichts der zunehmenden Anzahl an beschriebenen iatrogenen Infektionen im Rahmen medizinischer Eingriffe - auch dermatologischer - und der Vielfalt an möglichen klinischen Erscheinungsformen kommt atypischen Mykobakteriosen eine zunehmende differenzialdiagnostische Bedeutung zu. Diese stellen 
auch in Hinblick auf Sterilisation und ärztliche Hygiene erhöhte Anforderungen.

\section{Fallbericht}

\section{Anamnese}

Die 42-jährige Patientin stellte sich im Anschluss an einen Thailandaufenthalt dem gynäkologischen Notdienst erstmals im Juni 2000 mit Unterleibsschmerzen vor. Von diesem seien bei V.a. Mykose Itraconazol und Amoxicillin oral sowie topische Antimykotika verordnet worden. Wenige Wochen später befand sich die Patientin erneut in Thailand. Dort sei es zu einer weiteren Befundverschlechterung gekommen, so dass ein stationärer Krankenhausaufenthalt notwendig wurde, in dessen Rahmen eine Therapie mit Metronidazol erfolgt sei. Bei der Rückkehr nach Deutschland wurde ein Infekt mit Candida crusei diagnostiziert und mit Amphotericin oral und Econazol lokal therapiert. Im Verlauf erfolgte eine Vorstellung beim Hautarzt, der die Patientin mit V.a. Aktinomykose zu uns überwies. Eine Rhinokonjunktivitis allergica war bekannt, ansonsten keine relevanten Vorerkrankungen.

\section{Befund}

Bei Aufnahme fanden sich brettharte, bräunlich hyperpigmentierte, kutan-subkutane Knoten inguinal, perigenital und am Mons pubis (Abb. 1). Darin eingestreut zeigten sich multiple Fistelöffnungen mit spontaner Pusentleerung. Die Patientin beklagte ausgesprochene Druckschmerzhaftigkeit. Das übrige Integument sowie die Lymphknotenstationen waren unauffällig.

\section{Labor}

BKS 50/87 mm n. W., Leukozyten 11,7 Tsd/mic, Thrombozyten $551 \mathrm{Tsd} / \mathrm{mic}$, Neutrophile 73,5\%, Lymphozyten 17,0\%, CRP $0,62 \mathrm{mg} / \mathrm{dl}$.

Alle übrigen Laborparameter (Glucose, Harnstoff, Kreatinin, Harnsäure, Natrium, Kalium, GOT, GPT, LDH, AP, rGT, restliches Blutbild, PTT, Quick, INR, Triglyzeride, Cholesterin, Urinstatus, TPHA, HIV-Serologie) waren normwertig bzw. negativ.

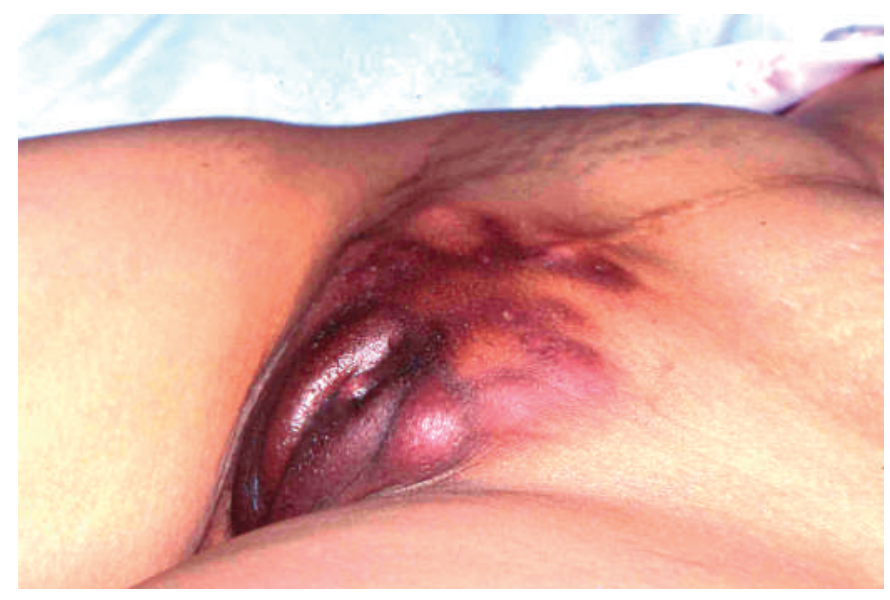

Abb. 1 Brettharte, druckschmerzhafte, kutan-subkutane Knoten in teils sporotrichoider Anordnung mit multiplen Fistelöffnungen.
Bei V.a. Aktinomykose und den Differenzialdiagnosen Tuberkulose und atypische Mykobakteriose erfolgte eine weitergehende Diagnostik.

Multitest Immignost: grenzwertig positiv für Tetanus, Diphtherie, negativ für Tuberkulin und andere Bakterien.

Mykologische Untersuchung vom Wundmaterial: vereinzelt Sporen, keine Drusen.

Sputum auf säurefeste Stäbchen: negativ $(2 \times$ kontrolliert $)$.

Mittelstrahlurin auf säurefeste Stäbchen: negativ ( $2 \times$ kontrolliert).

3 bakteriologische Abstriche vom Pus: initial zweimalig Mykobakterium chelonae, zuletzt aerob und anaerob kein Wachstum.

Bakteriologischer Abstrich von Wundmaterial: Staph. epidermidis.

Histologie vom Labium majorum rechts: Im Fett-Bindegewebe ausgedehnte, landkartenartig gestaltete, zellarme Nekrosefelder um die herum sich stellenweise ein lockerer Epitheloidzellwall entwickelt hat. Am äußeren Saum des z.T. breiten Epitheloidzellwalls in mäßiger Zahl Lymphozyten, auch einige Plasmazellen. Immer wieder mehrkernige histiozytäre Riesenzellen, wobei einzelne davon sich auch an zugrundegehendes Elasticamaterial anlagern.

Die Ziehl-Neelsen-Färbung verläuft negativ. Pilze sind nicht nachweisbar. Auch andere spezielle Erreger insbesondere Aktinomyzes-Drusen nicht nachweisbar.

Damit wird eine floride nekrotisierende granulomatöse Entzündung mit dem histologischen Bild einer Tuberkulose beschrieben.

Die weiteren paraklinischen Untersuchungen, Thoraxröntgen, Abdomensonographie und im Rahmen eines gynäkologischen Konsils durchgeführter, transvaginaler Ultraschall, waren, abgesehen von einer Hepatomegalie, unauffällig.

\section{Therapie und Verlauf}

Die stationäre Aufnahme erfolgte unter dem klinischen Verdacht einer Aktinomykose. Initial wurde hochdosiert mit täglich 30 Mio. IE Penicillin G i.v. behandelt. Topisch kam Ichthyol zum Einsatz.

Wir entließen die Patientin nach 4-wöchigem stationärem Aufenthalt mit rückläufigem Befund unter oraler Antibiose mit Cefuroxim $5002 \times 1$ und weiterer Lokaltherapie mit Ichthyol pur. Im Rahmen einer nachfolgenden ambulanten Wiedervorstellung zeigte sich eine anhaltende Befundbesserung. 
Mykobakterien zeichnen sich durch eine dicke, lipidreiche, säurefeste Kapsel aus, die sie sehr resistent gegen Umwelteinflüsse macht $[3,4,7-10]$.

Mykobakterien kommen im Wasser, in Staub, Schmutz, fauligen Abfällen oder im Tierreich $[11,12]$ vor. M. chelonae wurde in Leitungswasser [3,5], Aqua destillata, Gentianaviolettlösung und Methylenblau [13] in Krankenhäusern isoliert. Übertragungen wurden durch kontaminierte medizinische Instrumente (Venenverweilkanülen, Akupunkturnadeln [8,14], Insulinspritzen [15], Endoskopen $[4,10,16-18])$ und Bagatellverletzungen beschrieben.

Bei bis zu 18\% scheinbar Gesunder finden sich atypische Mykobakterien im Speichel und Sputum, in $1 / 33$ aller Fälle werden Primärdefekte als Ausgangspunkt der Infektion geschildert. Eine Übertragung von Mensch zu Mensch ist unwahrscheinlich. Etwa 90 Spezies sind bekannt, davon gelten etwa 30 als für den Menschen relevant [1].

Die Einteilung der Mykobakterien erfolgt in kultivierbare und nicht kultivierbare (Abb. 2), wie Mykobakterium leprae; in typische, wie M. tuberculosis und bovis, und atypische. Zur Einteilung der atypischen dient die Runyon-Klassifikation (Tab. 1), die nach der Wachstumsgeschwindigkeit und der Pigmentproduktion unterscheidet. Nur einzelne, wie M. ulcerans sind obligat pathogen, die meisten Vertreter sind opportunistische Keime, manche sind Saprophyten. Atypische Mykobakteriosen können sich als lokalisierte $[19,20]$ und auch als disseminierte Krankheitsbilder manifestieren. Disseminierte betreffen vor allem die Lunge $[1,21]$ und den Gastrointestinaltrakt und gehen mit Allgemeinsymptomen wie Fieber, Nachtschweiß, Gewichtsverlust und Diarrhöen einher.

Lokalisierte Formen betreffen vorwiegend die Haut oder die hautnahen, meist zervikalen Lymphknoten [1] und verursachen hier meist erythematopapulöse, teils warzige Läsionen, infiltrierte Knoten und Plaques wie im vorliegenden Fall, in ca. 20\% der Fälle in linearer, sporotrichoider Anordnung, sowie schlecht heilende oberflächliche und tiefe Ulzerationen. Teils finden sich Abszesse nach Injektionen [3,15], und die Zahl iatrogener Infekte steigt zusehend $[3-6,8,13,14,18,19-24]$.

Atypische Mykobakteriosen können vor allem bei Immunsuppression lymphogen und hämatogen streuen und führen bei Generalisation gewöhnlich zum Tode.

Histologisch finden sich teilweise verkäsende Granulome aus Epitheloid- und Fremdkörperriesenzellen mit lymphohistiozytärem und plasmazellulärem Umgebungsinfiltrat. Die Unterscheidung von den klassischen Mykobakteriosen ist auch in Spezialfärbungen schwierig. Bei immunsupprimierten Patienten kann aufgrund der verminderten zellulären Immunantwort das histologische Bild untypisch sein, mitunter finden sich nur diffuse Infiltrate.

Die Diagnose fußt auf der kulturellen Anzüchtung bei spezifischen Bedingungen aus Biopsie- und Abstrichmaterial; eine Re-

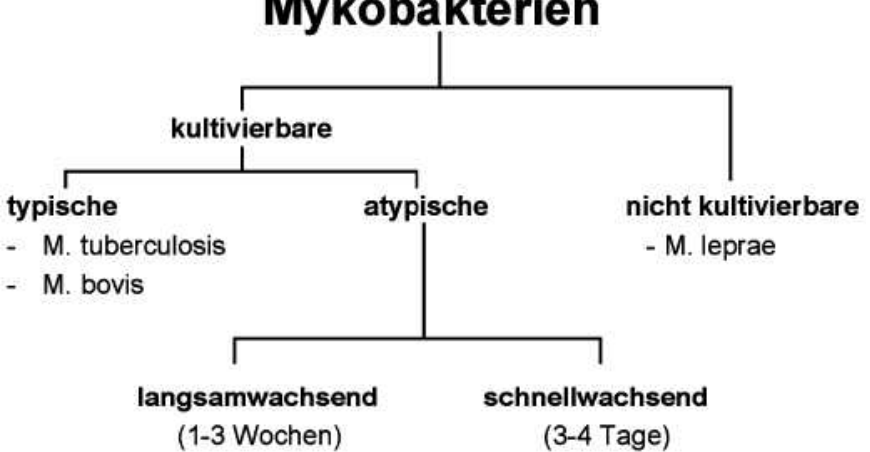

Abb. 2 Einteilung der Mykobakterien.

Tab. 1 Runyon-Klassifikation (Übersicht)

Runyon-Klassifikation atypischer Mykobakterien

I. Photochromogen (Pigmentproduktion bei Tageslicht und $37^{\circ} \mathrm{C}$ )

- M. kansaii

- M. marinum ${ }^{1}$ (Schwimmbadgranulom)

II. Skotochromogen (Pigmentproduktion ohne Licht bei $37^{\circ} \mathrm{C}$ )

- M. scrofulaceum

- M. szulgai

III. Nichtchromogen (keine Pigmentproduktion bei $37^{\circ} \mathrm{C}$ )

- M. avium-intracellulare-Komplex

- M. malmoense, M. xenopi

- M. ulcerans' (Buruli-Ulkus)

IV. Schnellwachsend (Kolonien binnen $3-4 d$ bei $37^{\circ} \mathrm{C}$ )

- M. fortuitum-chelonae-Komplex

- M. smegmatis (Saprophyt)

${ }^{1}$ Wachstumsoptimum bei $32^{\circ} \mathrm{C}$.

sistenzprüfung ist aufgrund unterschiedlicher und meist nur geringer Empfindlichkeit gegenüber den herkömmlichen Antibiotika therapeutisch indiziert. Auch gegen gebräuchliche Desinfektionsmaßnahmen wie Glutaraldehyd 2\% [4,10], Chlor [7] und Alkohol 75\% [3] zeigen Mykobakterien oft eine beharrliche Wiederstandsfähigkeit.

Das therapeutische Spektrum [2] reicht von der Exzision umschriebener Herde, der Monotherapie mit Trimethoprim/Sulfamethoxacol und Doxycyclin, einer Kombination von Amikacin und Clarithromycin bis hin zur kombinierten, an der Resistenzprüfung orientierten Antibiose und dem Einsatz der bewährten, klassischen antituberkulösen Dreiertherapie mit Isoniazid, Rifampicin und Pyrazinamid. Auch Erfolge mit Röntgenbestrahlung wurden beschrieben. In jedem Fall muss über die klinische Abheilung hinaus behandelt werden.

Angesichts der zunehmenden Anzahl an beschriebenen iatrogenen Infektionen im Rahmen medizinischer Eingriffe - auch dermatologischer - und der Vielfalt an möglichen klinischen Erscheinungsformen kommt atypischen Mykobakteriosen eine zunehmende differenzialdiagnostische Bedeutung zu. Diese stellen auch in Hinblick auf Sterilisation und ärztliche Hygiene erhöhte Anforderungen. 
Bei unserer Patientin gestaltete sich die endgültige Diagnosestellung schwierig. Anhand des klinischen Befundes wurden sowohl eine Hauttuberkulose als auch eine Aktinomykose diskutiert. Es konnten jedoch weder Drusen noch Mykobakterium tuberculosis nachgewiesen werden. Das einzig nachgewiesene Mykobakterium chelonae war vom Forschungszentrum Borstel, dem nationalen Referenzzentrum für Mykobakterien, als ohne klinische Bedeutung eingestuft worden. Daraufhin legten wir uns auf die Diagnose einer Aktinomykose fest, das Fehlen von Drusen interpretierten wir als Folge der prästationär erfolgten Antibiose. Rückblickend betrachtet, muss hierbei jedoch von einer Fehleinschätzung ausgegangen werden; die Vielzahl an vorliegenden Arbeiten und Kasuistiken belegt hinlänglich die klinische Relevanz vom Mykobakterium chelonae.

\section{Literatur}

${ }^{1}$ Katoch VM. Infections due to non-tuberculous mycobacteria (NTM). Indian J Med Res 2004; 120: 290-304

2 Braun-Falco O, Plewig G, Wolff HH. Dermatologie und Venerologie. 4. Auflage. Berlin, Heidelberg: Springer, 1997

${ }^{3}$ Tazawa S, Marumo K, Nakamura Y, Narushima M, Higuchi D. Evaluation of rapidly growing Mycobacteria isolates in a general hospital: reports from the hospital microbiology laboratory. Kekkaku 2001; 76: $419-426$

${ }^{4}$ Kressel AB, Kidd F. Pseudo-outbreak of Mycobacterium chelonae and Methylobacterium mesophilicum caused by contamination of an automated endoscopy washer. Infect Control Hosp Epidermiol 2001; 22: $414-418$

${ }^{5}$ Meyers H, Brown-Elliot BA, Moore D, Curry J, Truong C, Zhang Y, Wallace RJ, Jr. An outbreak of Mycobacterium chelonae infection following liposuction. Clin Infect Dis 2002; 34: 1500-1507

${ }^{6}$ Zhibang Y, Bi Xia Z, Qishan L, Lihao C, Xiangquan L, Huaping L. Largescale outbreak of infection with Mycobacterium chelonae subsp. abscessus after penicillin injection. J Clin Micobiol 2002; 40: 2626-2628

${ }^{7}$ Le Dantec C, Duguet JP, Montiel A, Dumoutir N, Dubrou S, Vincent V. Chlorine disinfection of atypical mycobacteria isolated from a water distribution system. Appl Environ Microbiol 2002; 68: 1025 - 1032

${ }^{8}$ Woo PC, Leung KW, Wong SS, Chong KT, Cheung EY, Yuen KY. Relatively alcohol-resistant mycobacteria are emerging pathogens in patients receiving acupuncture treatment. J Clin Microbiol 2002; 40: 12191224

${ }^{9}$ Yang SC, Hsueh PR, Lai HC, Teng LJ, Huang LM, Chen JM, Wang SK, Shie DC, Ho SW, Luh KT. High prevalence of antimicrobial resistance in rapidly growing mycobacteria in Taiwan. Antimicrob Agents Chemother 2003; 47: $1958-1962$
${ }^{10}$ Nomura K, Ogawa M, Chang B, Miyamoto H, Tanabe T, Tanigutchi H, Matsumoto T. Contamination of a bronchial fiberscope by mycobacteria linked to an automated bronchoscope disinfection machine. J UOEH 2000; 22: $159-165$

${ }^{11}$ Bercovier H, Vincent V. Mycobacterial infections in domestic and wild animals due to Mycobacterium marinum, M. fortuitum, M. chelonae, M. porcinum, M. farcinogenes, M. smegmatis, M. scrofulaceum, M. xenopi, M. kansaii, M. simiae and M. genavense. Rev Sci Tech 2001; 20 : $265-290$

12 Green SL, Lifland BD, Bouley DM, Brown BA, Wallace RJ, Ferrell JE. Disease attributed to Mycobacterium chelonae in South African clawed frogs (Xenopus laevis). Comp Med 2000; 50: 675-679

${ }^{13}$ Centers for Disease Control and Prevention (CDC). Mycobacterium chelonae infections associated with face lifts, New Jersey, 2002 - 2003. MMWR Morb Mortal Wkly Rep 2004; 53: 192 - 194

${ }^{14}$ Ara M, de Santamaria CS, Zaballos P, Yus C, Lezcano MA. Mycobacterium chelonae infection with multiple cutaneous lesions after treatment with acupuncture. Int J Dermatol 2003; 42: 642 - 644

${ }^{15}$ Finucane K, Ambrey P, Narayan S, Archer CB, Dayan C. Insulin injection abscesses caused by Mycobacterium chelonae. Diabetes Care 2003; 26: $2483-2484$

${ }^{16}$ Sethi NK, Aggarwal PK, Duggal L, Sachar VP. Mycobacterium chelonae infection following laparoscopic inguinal herniorrhaphy. J Assoc Physicians India 2003; $51: 81-82$

${ }^{17}$ Gillespie TG, Hogg L, Budge E, Duncan A, Coia JE. Mycobacterium chelonae isolated from rinse water within an endoscope washer-disinfector. J Hosp Infect 2000; 45: 332 - 334

${ }^{18}$ Rodrigues C, Mehta A, Jha U, Bharachua M, Dastur FD, Udwadia TE. Nosocomial Mycobacterium chelonae infection in laparoscopic surgery. Infect Control Hosp Epidermiol 2001; 22: 474-475

${ }^{19}$ Scott IU, Lieb DF, Flynn HW, Dessouki A, Murray TG, Miller D. Endophthalmitis caused by Mycobacterium chelonae: selection of antibiotics and outcomes of treatment. Arch Ophthalmol 2003; 121: 573-576

${ }^{20}$ Gollwitzer H, Langer R, Diehl P, Mittelmeier W. Chronic osteomyelitis due to Mycobacterium chelonae diagnosed by polymerase chain reaction homology matching. A case report. J Bone Joint Surg Am 2004; 86: $1296-1301$

${ }^{21}$ Hazelton TR, Newell JD, Cook JL, Huitt GA, Lynch DA. CT findings in 14 patients with Mycobacterium chelonae pulmonary infection. AJR Am J Roentgenol 2000; 175: $413-416$

${ }^{22}$ Malecha MA, Doughman DJ. Mycobacterium chelonae keratitis associated with soft contact lens water. CLAO J 2002; 28: 228-230

${ }^{23}$ Sniezek PJ, Graham BS, Busch HB, Lederman ER, Lim ML, Poggemyer K, Kao A, Mizrahi M, Washabaugh G, Yakrus M, Winthrop K. Rapidly growing mycobacterial infections after pedicures. Arch Dermatol 2003; 139: 629-634

${ }^{24}$ Hassler D, Braun R. Wer schön sein will muss manchmal leiden: Mykobakteriose nach Schönheitsoperation in der Karibik. Dtsch Med Wschr 2004; 129: 1668 